TRANSACTIONS OF THE

AMERICAN MATHEMATICAL SOCIETY

Volume 354, Number 3, Pages 1117-1135

S 0002-9947(01)02894-X

Article electronically published on October 4, 2001

\title{
ON A STOCHASTIC NONLINEAR EQUATION IN ONE-DIMENSIONAL VISCOELASTICITY
}

\author{
JONG UHN KIM
}

\begin{abstract}
In this paper we discuss an initial-boundary value problem for a stochastic nonlinear equation arising in one-dimensional viscoelasticity. We propose to use a new direct method to obtain a solution. This method is expected to be applicable to a broad class of nonlinear stochastic partial differential equations.
\end{abstract}

\section{INTRODUCTION}

In this paper we will discuss an initial-boundary value problem associated with one-dimensional viscoelasticity of rate type with random force. The equation is of the following form:

$$
u_{t t}=\eta\left(u_{x}, u_{t x}\right)_{x}+\xi+f,
$$

where $u(t, x)$ is the displacement, $\eta$ is the stress function depending on the deformation gradient $u_{x}$ and its time derivative $u_{t x}, \xi$ is a random perturbation depending on $u_{t x}$, and $f$ represents a given random body force. When $\xi \equiv 0, f$ is deterministic and $\eta$ is linear in $u_{t x}$, solutions were obtained by Greenberg, MacCamy and Mizel [8] and Andrews [1]. When $\eta$ is nonlinear in $u_{t x}$, Dafermos [4] proved the existence and uniqueness of a solution. Here the principal part of the equation is patterned after the equation discussed in [4], but with random force. There are many known results on semi-linear stochastic evolution equations. In particular, semi-linear parabolic equations have been extensively investigated. See references in Da Prato and Zabczyk [5]. The major tool in this case is the stochastic convolution. This approach was also adopted in de Bouard and Debussche [6], and Printems [13. For quasi-linear stochastic equations, only a particular class of equations has been analyzed. The main reason is that we do not have compactness of approximate solutions which ensures strong convergence, which is crucial to handle the nonlinearity. Let us elaborate on this. For deterministic equations, we typically obtain uniform estimates for a sequence of approximate solutions in a Banach space $\mathcal{X}$ which is compactly imbedded into another Banach space $\mathcal{Y}$. Then, we can extract a subsequence which converges strongly in $\mathcal{Y}$. In case of a stochastic version of the same equation, typical estimates are obtained in the space $L^{p}(\Omega ; \mathcal{X})$ where $\Omega$ is a probability space. Unfortunately, the embedding $L^{p}(\Omega ; \mathcal{X}) \rightarrow L^{p}(\Omega ; \mathcal{Y})$ is not compact. But if the property of a monotone operator can be used, then weak convergence is enough to obtain a solution by Minty's method. This is exactly the

Received by the editors October 19, 2000 and, in revised form, May 4, 2001.

2000 Mathematics Subject Classification. Primary 35R60, 60H15, 74D10.

Key words and phrases. Viscoelasticity, random force, white noise, pathwise solutions. 
case with the class of quasi-linear stochastic equations that has been analyzed in the past. We will briefly review what has been known. The general form of an equation in this class is

$$
v_{t}=A(t, v)+f
$$

where $f$ is random. To formulate the conditions on the nonlinear term $A(t, v)$, we consider a Hilbert space $H$, and a Banach space $V$ and its dual $V^{\prime}$ such that there is a continuous injection: $V \rightarrow H \rightarrow V^{\prime}$. The typical assumptions on $A$ are as follows.

(i) For some constants $M>0$, and $p \geq 2,\|A(t, v)\|_{V^{\prime}} \leq M\|v\|_{V}^{p-1}$ holds for all $t$ and $v \in V$.

(ii) For all $t$ and $v_{1}, v_{2} \in V,\left\langle A\left(t, v_{1}\right)-A\left(t, v_{2}\right), v_{1}-v_{2}\right\rangle_{V^{\prime}, V} \leq 0$ holds.

(iii) For some positive constants $M$ and $\lambda,\langle A(t, v), v\rangle_{V^{\prime}, V} \leq-M\|v\|_{V}^{p}+\lambda|v|_{H}^{2}$ holds for all $v \in V$ and all $t$.

(iv) For each $t$, the operator $A(t, \cdot)$ is hemicontinuous from $V$ into $V^{\prime}$, and for each $v \in V, A(\cdot, v)$ is strongly measurable.

Here $\langle\cdot, \cdot\rangle_{V^{\prime}, V}$ is the duality pairing between $V^{\prime}$ and $V$. When $f$ is deterministic, a prototype for quasi-linear equations was fully investigated in Lions [9]. Stochastic versions were discussed in Benssousan and Temam [2], Métivier and Pistone [10], Métivier and Viot [11, and Pardoux [12. The above conditions were essentially used. Here the condition (i) is a growth condition and $p$ is usually larger than 2. The condition (ii) requires that $A$ is monotone. It can be a little relaxed by considering $v e^{\lambda t}$ in place of $v$ in (0.2). The coercive condition (iii) combined with (i) imposes a severe restriction on the form of $A(t, v)$. However, if sufficient a priori estimates can be obtained without (i) or (iii), the argument of Minty's method can be still used only by (ii) and (iv). Nevertheless this monotonicity condition can be essentially broken by various random nonlinear terms of lower order. In such cases, Minty's method cannot be used. The particular equation discussed in this work is such an example.

The main goal of this work is to find an alternative method to overcome the difficulty due to the lack of compactness explained above. Perhaps one alternative is first to obtain a uniform estimate of approximate solutions in $L^{p}(\Omega ; \mathcal{X})$, and construct a martingale solution with values in $\mathcal{Y}$ via the Skorokhod theorem, where $\mathcal{X}$ is compactly embedded into $\mathcal{Y}$. Then, the pathwise uniqueness yields a solution of the original problem with values in $\mathcal{Y}$. This highy nontrivial result was proved by Yamada and Watanabe [18, and the procedure was extended to stochastic partial differential equations by Viot [16]. This approach was used for a semilinear heat equation by Viot 14, and for the stochastic KdV equation by Printems [13.

Here we propose to use a more direct method to construct solutions. As usual, we first set up a scheme of Galerkin approximation and obtain a priori estimates. But the method of constructing a pathwise solution is entirely different from all the previous methods. We use a very simple measure-theoretic argument combined with the pathwise uniqueness. This approach bypasses martingale solutions and dispenses with other heavy machinery. Furthermore, the solution has values in the smaller space $\mathcal{X}$ in the above setting. Our method is expected to have applications to many other nonlinear stochastic partial differential equations. The problem will be formulated and the main result will be presented in Section 1, and the technical proof will be given in Section 2 . 


\section{Formulation of the PRoblem AND the Main RESUlt}

We will follow the stochastic functional setting in Walsh [17]. Let $\mathcal{B}([0, \infty) \times[0,1])$ be the set of all Borel subsets of $[0, \infty) \times[0,1]$, and $\mu$ be the Lebesgue measure over $R^{2}$. Let $\{W(h)\}$ with $h \in L^{2}([0, \infty) \times[0,1], \mathcal{B}, \mu)$ be a centered Gaussian family of random variables on a complete probability space $(\Omega, \mathcal{F}, P)$ such that the covariance is given by

$$
E(W(g) W(h))=\langle g, h\rangle_{L^{2}([0, \infty) \times[0,1], \mathcal{B}, \mu)} .
$$

Let $\left\{\mathcal{F}_{t}\right\}_{t>0}$ be a right-continuous filtration on $(\Omega, \mathcal{F}, P)$ such that $\mathcal{F}_{0}$ contains all $P$-negligible sets and $W\left(\chi_{[0, t] \times A}\right)$ is $\mathcal{F}_{t}$-measurable for each $A \in \mathcal{B}([0,1])$ and $t \geq 0$. Here $\chi_{[0, t] \times A}$ is the characteristic function of the set $[0, t] \times A$. Then, $\left(\Omega, \mathcal{F}, \mathcal{F}_{t}, P\right)$ is our stochastic basis and $W$ is the white noise. A Brownian sheet is defined by

$$
B(t, x)=W\left(\chi_{[0, t] \times[0, x]}\right) .
$$

We assume that the random force $f$ in $(0.1)$ is given by

$$
f=\Phi \frac{\partial^{2} B(t, x)}{\partial t \partial x}
$$

where $\Phi$ is a Hilbert-Schmidt operator from $L^{2}(0,1)$ into $H^{1}(0,1)$. Similar random force was also used in de Bouard and Debussche [6]. An example of such a force is

$$
f=\sum_{j=1}^{N} c_{j}(x) \frac{d}{d t} b_{j}(t),
$$

where each $c_{j} \in H^{1}(0,1)$ and $b_{j}$ 's are the standard Brownian motions which are mutually independent and adapted to $\left\{\mathcal{F}_{t}\right\}$. Throughout this paper, we denote by $H^{s}(0,1)$ the usual Sobolev space of order $s$ over the open interval $(0,1)$. We also make the following assumptions on $\eta$.

[I] $\quad \eta \in C^{1}\left(R^{2}\right)$ and $\eta(0,0)=0$ with the following properties: for some positive constants $M_{1}, M_{2}$ and $M_{3}$,

$$
\begin{gathered}
\eta_{q}(p, q) \geq M_{1}, \quad \text { for all } p, q, \\
\left|\eta_{p}(p, q)\right| \leq M_{2} \sqrt{\eta_{q}(p, q)}, \quad \text { for all } p, q,
\end{gathered}
$$

and

$$
|\eta(p, q)| \leq M_{3}(1+|p|+|q|)^{5}, \quad \text { for all } p, q,
$$

where $\eta_{p}=\frac{\partial}{\partial p} \eta$, and $\eta_{q}=\frac{\partial}{\partial q} \eta$. The assumptions on $\xi=\xi(t, \omega, h)$ are as follows.

[II] For each $t \geq 0$ and $h \in R, \xi(t, \omega, h)$ is $\mathcal{F}_{t}$-measurable.

[III] For $P$-almost all $\omega \in \Omega, \xi(t, \omega, h)$ and $\frac{\partial}{\partial h} \xi(t, \omega, h)$ are continuous in $(t, h)$ such that

$$
\xi(t, \omega, 0) \in L^{1}\left(\Omega ; L^{\infty}(0, \infty)\right), \quad \frac{\partial \xi}{\partial h}(t, \omega, h) \in L^{6 / 5}\left(\Omega ; L^{\infty}((0, \infty) \times R)\right) .
$$

We formulate the initial-boundary value problem as follows.

$$
\begin{gathered}
u_{t t}=\eta\left(u_{x}, u_{t x}\right)_{x}+\xi\left(t, \omega, u_{t x}\right)+\Phi \frac{\partial^{2} B(t, x)}{\partial t \partial x}, \quad \text { for } 0<x<1, t>0, \\
u_{x}=0, \quad \text { at } x=0,1, \quad \text { for } t>0
\end{gathered}
$$




$$
u=\hat{u}_{0}, \quad u_{t}=\hat{u}_{1}, \quad \text { at } t=0, \quad \text { for } 0<x<1 .
$$

Here (1.10) is the traction-free boundary conditions, because $\eta(0,0)=0$.

We adopt the following definition of a solution to (1.9) - (1.11).

Definition 1.1. Let $T>0$ be given. A $H^{1}(0,1)$-valued continuous process $u$ adapted to $\left\{\mathcal{F}_{t}\right\}$ is called a solution of $(1.9)-(1.11)$ if $u_{t x} \in L^{\infty}\left(0, T ; L^{2}(0,1)\right) \cap$ $L^{2}\left(0, T ; H_{0}^{1}(0,1)\right) \cap L^{6}\left(0, T ; H^{1 / 3}(0,1)\right)$, for $P$-almost all $\omega \in \Omega$, and if for each $\phi \in C^{\infty}([0,1])$ with $\phi_{x}(0)=\phi_{x}(1)=0$,

$$
\begin{aligned}
\left\langle u_{t}(t)\right. & \left.-\hat{u}_{1}, \phi\right\rangle=-\int_{0}^{t} \int_{0}^{1} \eta\left(u_{x}, u_{s x}\right) \phi_{x} d x d s \\
& +\int_{0}^{t} \int_{0}^{1} \xi\left(s, \omega, u_{s x}\right) \phi d x d s+\int_{0}^{t} \int_{0}^{1} \Phi^{\star} \phi d W
\end{aligned}
$$

holds for all $t \in[0, T]$, for $P$-almost all $\omega \in \Omega$, and $u(0)=\hat{u}_{0}$ holds for $P$-almost all $\omega \in \Omega$.

Here, $\langle\cdot, \cdot\rangle$ denotes the $L^{2}(0,1)$-product, and $\Phi^{\star}$ is the adjoint of $\Phi$, i.e., for all $g \in L^{2}(0,1)$ and $h \in H^{1}(0,1)^{*},\langle\Phi g, h\rangle_{H^{1}, H^{1 *}}=\left\langle g, \Phi^{\star} h\right\rangle$, where the bracket in the left-hand side is the duality pairing between $H^{1}(0,1)$ and $H^{1}(0,1)^{*}$, which is the dual of $H^{1}(0,1)$. In particular, when $h \in L^{2}(0,1)$, it holds that $\langle\Phi g, h\rangle_{H^{1}, H^{1 *}}=$ $\langle\Phi g, h\rangle$. Since $\Phi$ is a Hilbert-Schmidt operator from $L^{2}(0,1)$ into $H^{1}(0,1)$, its operator norm is given by

$$
\begin{aligned}
\|\Phi\| & =\left(\sum_{k=0}^{\infty} \sum_{j=1}^{\infty} \lambda_{j}\left|\left\langle\Phi\left(e_{k}\right), e_{j}\right\rangle\right|^{2}+\sum_{k=0}^{\infty}\left|\left\langle\Phi\left(e_{k}\right), e_{0}\right\rangle\right|^{2}\right)^{1 / 2} \\
& =\left(\sum_{k=0}^{\infty} \sum_{j=1}^{\infty} \lambda_{j}\left|\left\langle e_{k}, \Phi^{\star}\left(e_{j}\right)\right\rangle\right|^{2}+\sum_{k=0}^{\infty}\left|\left\langle e_{k}, \Phi^{\star}\left(e_{0}\right)\right\rangle\right|^{2}\right)^{1 / 2}<\infty,
\end{aligned}
$$

where $\lambda_{j}$ 's and $e_{j}$ 's are defined by (2.2) below.

In the meantime, $d W$ stands for the orthogonal martingale measure induced by the white noise above. Under the above assumptions, our main result is the following.

Theorem 1.2. Suppose that $\hat{u}_{0}$ is $\tilde{H}^{2}(0,1)$-valued $\mathcal{F}_{0}$-measurable and $\hat{u}_{1}$ is $H^{1}(0,1)$ valued $\mathcal{F}_{0}$-measurable such that $\hat{u}_{0} \in L^{6}\left(\Omega ; \tilde{H}^{2}(0,1)\right)$ and $\hat{u}_{1} \in L^{6}\left(\Omega ; H^{1}(0,1)\right)$. Then, for any given $T>0$, there is a unique solution of (1.9) - (1.11).

Here we use the notation

$$
\tilde{H}^{2}(0,1)=\left\{f \mid f, f_{x}, f_{x x} \in L^{2}(0,1) \text { and } f_{x}(0)=f_{x}(1)=0\right\} .
$$

The solution is said to be unique if any two solutions are indistinguishable. The proof will be presented in the next section.

\section{Proof of the MAIN RESUlt}

We outline the general strategy. First we set up an approximation scheme using a special orthonormal basis for $L^{2}(0,1)$. We then derive basic a priori estimates. Finally, a pathwise solution is constructed, and necessary properties for the solution are verified. 
2.1. Approximation and a priori estimates. We choose a complete orthonormal basis $\left\{e_{k}\right\}_{k=0}^{\infty}$ for $L^{2}(0,1)$ from the eigenvalue problem

$$
\left\{\begin{array}{l}
-\nabla^{2} e_{k}=\lambda_{k} e_{k}, \\
\nabla e_{k}(0)=\nabla e_{k}(1)=0 .
\end{array}\right.
$$

Here $\nabla=\frac{d}{d x}$. In fact, we have

(2.2) $\quad \lambda_{0}=0, \quad e_{0}(x)=1, \quad \lambda_{k}=k^{2} \pi^{2}, \quad e_{k}(x)=\sqrt{2} \cos (k \pi x), \quad k=1,2, \cdots$.

Next we approximate $\xi$ by using (1.8). Let us write

$$
\zeta_{N}(h)=\left\{\begin{array}{l}
h, \quad \text { for }|h| \leq N, \\
N \operatorname{sign}(h), \quad \text { for }|h|>N,
\end{array}\right.
$$

and define

$$
\xi_{N}(t, \omega, h)=\zeta_{N}(\xi(t, \omega, 0))+\int_{0}^{h} \zeta_{N}\left(\frac{\partial \xi}{\partial y}(t, \omega, y)\right) d y
$$

We then consider the Galerkin approximation. Let us write for each $m=1,2, \cdots$,

$$
u_{m}(t, x, \omega)=\sum_{k=0}^{m} d_{m k}(t, \omega) e_{k}(x),
$$

and

$$
c_{m k}(t, \omega)=\partial_{t} d_{m k}(t, \omega) .
$$

We fix $m \geq 1$, and discuss the following system of stochastic integral equations.

$$
\begin{aligned}
& c_{m k}(t)-\left\langle\hat{u}_{1}, e_{k}\right\rangle=-\int_{0}^{t} \int_{0}^{1} \eta\left(\sum_{j=1}^{m} d_{m j} \nabla e_{j}, \sum_{j=1}^{m} c_{m j} \nabla e_{j}\right) \nabla e_{k} d x d s \\
&+\int_{0}^{t} \int_{0}^{1} \xi_{m}\left(s, \omega, \sum_{j=1}^{m} c_{m j} \nabla e_{j}\right) e_{k} d x d s \\
&+\int_{0}^{t} \int_{0}^{1} \Phi^{\star} e_{k} d W, \quad t \in[0, T], \quad k=0, \cdots, m, \\
& d_{m k}(t)=\left\langle\hat{u}_{0}, e_{k}\right\rangle+\int_{0}^{t} c_{m k}(s) d s, \quad t \in[0, T], \quad k=0, \cdots, m .
\end{aligned}
$$

For a priori estimates, we first note that for some positive constants $K_{1}, K_{2}$,

$$
\begin{array}{r}
-\left(\eta\left(p_{1}, q_{1}\right)-\eta\left(p_{2}, q_{2}\right)\right)\left(q_{1}-q_{2}\right) \leq-K_{1}\left(q_{1}-q_{2}\right)^{2}+K_{2}\left(p_{1}-p_{2}\right)^{2}, \\
\text { for all }\left(p_{1}, q_{1}\right),\left(p_{2}, q_{2}\right) \in R^{2},
\end{array}
$$

which follows from (1.5), (1.6) and the mean value theorem. We will also use the fact that

$$
B_{j}(t)=\int_{0}^{t} \int_{0}^{1} \Phi^{\star} e_{j} d W
$$

is a continuous martingale adapted to $\mathcal{F}_{t}$. It follows from the Burkholder-DavisGundy inequalities that

$$
E\left(\sup _{0 \leq t \leq T}\left|B_{j}(t)\right|^{p}\right) \leq C_{p} E\left(\left(\int_{0}^{T} \int_{0}^{1}\left|\Phi^{\star} e_{j}\right|^{2} d x d t\right)^{p / 2}\right)
$$


for some positive constant $C_{p}$ depending only on $p>1$. Since the function $\eta$ may not grow linearly in its arguments, the standard result on the existence of solutions for stochastic differential equations cannot be applied. But a result of Métivier and Pistone [10] can be used. For this, let us introduce a $R^{2 m}$-valued random function $X(t)$ whose components are defined by

$$
\left\{\begin{array}{l}
X_{k}(t)=e^{-\lambda t}\left(c_{m k}(t)-\int_{0}^{t} \int_{0}^{1} \Phi^{\star} e_{k} d W\right), \quad k=1, \cdots, m, \\
X_{m+k}(t)=d_{m k}(t) e^{-\lambda t}, \quad k=1, \cdots, m
\end{array}\right.
$$

The above system (2.7) and (2.8) for $1 \leq k \leq m$ can be put in the form

$$
X(t, \omega)=Y(\omega)+\int_{0}^{t} A_{\lambda}(s, \omega, X(s, \omega)) d s,
$$

where the $R^{2 m}$-valued function $A_{\lambda}$ is defined as follows. For $1 \leq k \leq m$, the $k$-th component of $A_{\lambda}(t, \omega, X(t, \omega))$ is

$$
\begin{aligned}
& -\int_{0}^{1} e^{-\lambda t} \eta\left(\sum_{j=1}^{m} e^{\lambda t} X_{m+j} \nabla e_{j}, \sum_{j=1}^{m}\left(e^{\lambda t} X_{j}+\int_{0}^{t} \int_{0}^{1} \Phi^{\star} e_{j} d W\right) \nabla e_{j}\right) \nabla e_{k} d x \\
& +\int_{0}^{1} e^{-\lambda t} \xi_{m}\left(t, \omega, \sum_{j=1}^{m}\left(e^{\lambda t} X_{j}+\int_{0}^{t} \int_{0}^{1} \Phi^{\star} e_{j} d W\right) \nabla e_{j}\right) e_{k} d x-\lambda X_{k}
\end{aligned}
$$

and the $(m+k)$-th component is

$$
-\lambda X_{m+k}+X_{k}+e^{-\lambda t} \int_{0}^{t} \int_{0}^{1} \Phi^{\star} e_{k} d W .
$$

The components of the initial value $Y$ are given by

$$
Y_{k}=\left\langle\hat{u}_{1}, e_{k}\right\rangle \quad \text { and } \quad Y_{m+k}=\left\langle\hat{u}_{0}, e_{k}\right\rangle, \quad 1 \leq k \leq m .
$$

It is apparent that $Y$ is $\mathcal{F}_{0}$-measurable and belongs to $L^{6}(\Omega)$. It is also obvious that for each $\lambda \geq 0$ and each $h \in R^{2 m}, A_{\lambda}(t, \omega, h)$ is a predictable process, i.e., measurable with respect to the $\sigma$-algebra generated by all continuous adapted processes. By virtue of $(2.3),(2.4),(2.9)$ and the fact that $B_{j}(t)$ is continuous for $P$-almost all $\omega$, we can choose a positive number $\lambda$ depending only on $K_{1}, K_{2}$ and $m$, and find a subset $\tilde{\Omega}$ with $P(\tilde{\Omega})=1$ such that for each $t \in[0, T]$ and each $\omega \in \tilde{\Omega}$, $A_{\lambda}(t, \omega, h)$ is continuous in $h$, and for all $h_{1}, h_{2} \in R^{2 m}$,

$$
\left\langle A_{\lambda}\left(t, \omega, h_{1}\right)-A_{\lambda}\left(t, \omega, h_{2}\right), h_{1}-h_{2}\right\rangle_{R^{2 m}} \leq 0 .
$$

Furthermore, it follows from (1.7), (2.4) and (2.11) that for each $L>0$, there is a predictable process $\phi_{L}(t, \omega)$ such that

$$
\left|A_{\lambda}(t, \omega, h)\right| \leq \phi_{L}(t, \omega), \quad \text { for all } h \in R^{2 m} \text { with }|h| \leq L
$$

and

$$
\phi_{L} \in L^{6}(\Omega ; C([0, T])) .
$$

According to the result of [10], we have

Lemma 2.1. There is a unique continuous adapted process $X$ which satisfies (2.13) on the interval $[0, T]$, for $P$-almost all $\omega \in \Omega$. Furthermore, $X(t, \omega)$ is $\mathcal{F}_{t}$-measurable and belongs to $L^{6}(\Omega ; C([0, T]))$. 
The last assertion follows easily from (2.13) and (2.17) - (2.19). We now have a solution of $(2.7)$ and $(2.8)$ on the interval $[0, T]$. Here $c_{m 0}$ is determined by $c_{m k}, k=1, \cdots, m$, through (2.7).

For later use, we will clarify measurability of $c_{m k}$ 's. We first define our terminology.

Definition 2.2. Let $\mathcal{U}$ be a Banach space and $\mathcal{G}$ be a $\sigma$-algebra over $\Omega$. A function $g: \Omega \rightarrow \mathcal{U}$ is said to be $\mathcal{U}$-valued $\mathcal{G}$-measurable if $g^{-1}(V) \in \mathcal{G}$ for every open subset $V$ of $\mathcal{U}$.

Lemma 2.3. Fix any $t^{*} \in(0, T]$. Each $c_{m k}$ is $C\left(\left[0, t^{*}\right]\right)$-valued $\mathcal{F}_{t^{*}-\text { measurable, }}$ and also, $H^{1 / 4}\left(0, t^{*}\right)$-valued $\mathcal{F}_{t^{*}-\text { measurable. }}$

Proof. Let us fix $m \geq 1$ and $k \geq 1$, and define

$$
q_{N}(t)=c_{m k}(0) \chi_{\{0\}}+\sum_{j=1}^{N} c_{m k}\left(t_{j-1}\right) \chi_{\left(t_{j-1}, t_{j}\right]}(t),
$$

where $0=t_{0}<t_{1}<\cdots<t_{N}=t^{*}, t_{j}-t_{j-1}=t^{*} / N$, and $\chi$ stands for a characteristic function. Since $c_{m j}$ is a continuous process adapted to $\left\{\mathcal{F}_{t}\right\}$, each $q_{N}$ is $L^{\infty}\left(0, t^{*}\right)$-valued $\mathcal{F}_{t^{*}}$-measurable. In the meantime, for $P$-almost all $\omega$,

$$
\left\|q_{N}-c_{m k}\right\|_{L^{\infty}\left(0, t^{*}\right)} \rightarrow 0, \quad \text { as } N \rightarrow \infty
$$

Since $\mathcal{F}_{0}$ contains all $P$-negligible sets, $c_{m k}$ is $L^{\infty}\left(0, t^{*}\right)$-valued $\mathcal{F}_{t^{*}}$-measurable. But for $P$-almost all $\omega, c_{m k}$ is continuous in $t$, and $C\left(\left[0, t^{*}\right]\right)$ is a closed subspace

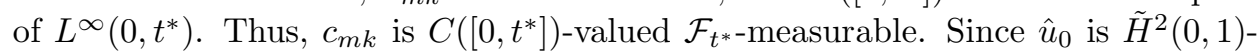
valued $\mathcal{F}_{0}$-measurable and $\hat{u}_{0} \in L^{6}\left(\Omega, \tilde{H}^{2}(0,1)\right)$, it follows from $(2.8)$ that $d_{m k}$ is also $C\left(\left[0, t^{*}\right]\right)$-valued $\mathcal{F}_{t^{*}}$-measurable. Next we consider (2.7) and (2.8). For fixed $\omega$, the mapping

$\left(d_{m 1}, \cdots, d_{m m}, c_{m 1}, \cdots, c_{m m}\right) \mapsto-\int_{0}^{(\cdot)} \int_{0}^{1} \eta\left(\sum_{j=1}^{m} d_{m j} \nabla e_{j}, \sum_{j=1}^{m} c_{m j} \nabla e_{j}\right) \nabla e_{k} d x d s$

is continuous from $\left(C\left(\left[0, t^{*}\right]\right)\right)^{2 m}$ into $H^{1}\left(0, t^{*}\right)$, for each $k=1, \cdots, m$. Since $c_{m k}$ 's

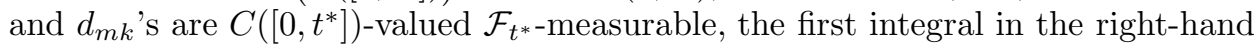

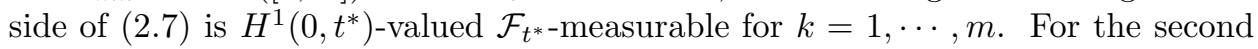
integral in (2.7), we need more work. The integral

$$
\int_{0}^{1} \xi_{m}\left(t, \omega, \sum_{j=1}^{m} h_{j}(t) \nabla e_{j}\right) e_{k} d x
$$

is continuous in $t$ for $P$-almost all $\omega$, and is adapted to $\left\{\mathcal{F}_{t}\right\}$ if each deterministic $h_{j}(t)$ is continuous in $t$. Hence, by the same argument as above, it is $C\left(\left[0, t^{*}\right]\right)$ valued $\mathcal{F}_{t^{*}}$-measurable if $h_{j} \in C\left(\left[0, t^{*}\right]\right), j=1, \cdots, m$. In the meantime, the mapping

$$
g \mapsto \int_{0}^{(\cdot)} g(s) d s
$$


is continuous from $C\left(\left[0, t^{*}\right]\right)$ into $H^{1}\left(0, t^{*}\right)$. Therefore, the function $Q(\omega, h)$ defined by

$$
Q(\omega, h)(t)=\int_{0}^{t} \int_{0}^{1} \xi_{m}\left(t, \omega, \sum_{j=1}^{m} h_{j}(t) \nabla e_{j}\right) e_{k} d x d s
$$

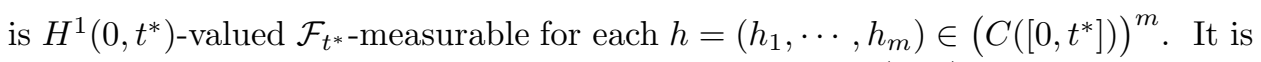
easy to see that for $P$-almost all $\omega$, the mapping $h \mapsto Q(\omega, h)$ is continuous from $\left(C\left(\left[0, t^{*}\right]\right)\right)^{m}$ to $H^{1}\left(0, t^{*}\right)$. Since $\left(C\left(\left[0, t^{*}\right]\right)\right)^{m}$ is a separable Banach space, each $\left(C\left(\left[0, t^{*}\right]\right)\right)^{m}$-valued $\mathcal{F}_{t^{*}}$-measurable function $\psi$ can be approximated by a sequence of simple functions $\left\{\phi_{N}\right\}_{N=1}^{\infty}$ of the form

$$
\phi_{N}(t, \omega)=\sum_{k=1}^{N} g_{N, k}(t) \chi_{N, k}(\omega)
$$

where each $g_{N, k} \in\left(C\left(\left[0, t^{*}\right]\right)\right)^{m}$, and each $\chi_{N, k}$ is a $\mathcal{F}_{t^{*}}$-measurable characteristic function such that $\chi_{N, k}(\omega) \chi_{N, j}(\omega)=0$, for $j \neq k$ and $\sum_{k=1}^{N} \chi_{N, k}(\omega)=1$, for all $\omega$. Here $\phi_{N}$ converges to $\psi$ in $\left(C\left(\left[0, t^{*}\right]\right)\right)^{m}$ as $N \rightarrow \infty$, for each $\omega$. Now we note that

$$
Q_{N}=Q\left(\omega, \phi_{N}\right)=\sum_{k=1}^{N} Q\left(\omega, g_{N, k}(t)\right) \chi_{N, k}(\omega)
$$

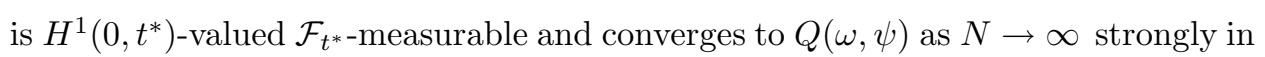
$H^{1}\left(0, t^{*}\right)$ for $P$-almost all $\omega$. Consequently, the second integral in $(2.7)$ is $H^{1}\left(0, t^{*}\right)$ valued $\mathcal{F}_{t^{*}}$-measurable. Next we rewrite $(2.10)$ :

$$
B_{k}(t)=\int_{0}^{t} \int_{0}^{1} \Phi^{*} e_{k} d W, \quad k=0, \cdots, m
$$

Since $B_{k}(t)$ is a continuous martingale adapted to $\left\{\mathcal{F}_{t}\right\}$, it is $\mathcal{B}\left(\left[0, t^{*}\right]\right) \otimes \mathcal{F}_{t^{*}}$ measurable as a function on $\left[0, t^{*}\right] \times \Omega$, where $\mathcal{B}\left(\left[0, t^{*}\right]\right)$ is the Borel $\sigma$-algebra over $\left[0, t^{*}\right]$. It is easy to see that

$$
E\left(\int_{0}^{t^{*}} \int_{0}^{t^{*}} \frac{\left|B_{k}\left(t_{2}\right)-B_{k}\left(t_{1}\right)\right|^{2}}{\left|t_{2}-t_{1}\right|^{1+1 / 2}} d t_{1} d t_{2}\right) \leq M
$$

for some positive constant $M$ independent of $t^{*}$ and $k$. This implies that for $P$ almost all $\omega, B_{k}$ is $H^{1 / 4}\left(0, t^{*}\right)$-valued. Let

$$
\gamma_{N}(t)=\sum_{j=1}^{N}\left(2 / t^{*}\right)\left(\int_{0}^{t^{*}} B_{k}(s) \sin \left(j \pi s / t^{*}\right) d s\right) \sin \left(j \pi t / t^{*}\right) .
$$

Since $B_{k}$ is $\mathcal{B}\left(\left[0, t^{*}\right]\right) \otimes \mathcal{F}_{t^{*}}$-measurable, the integral term in $(2.28)$ is $\mathcal{F}_{t^{*}}$-measurable, and hence, $\gamma_{N}$ is $H^{1 / 4}\left(0, t^{*}\right)$-valued $\mathcal{F}_{t^{*}}$-measurable. Meanwhile, for $P$-almost all $\omega, \gamma_{N}$ converges strongly to $B_{k}$ in $H^{1 / 4}\left(0, t^{*}\right)$ as $N \rightarrow \infty$. Consequently, $B_{k}$ is $H^{1 / 4}\left(0, t^{*}\right)$-valued $\mathcal{F}_{t^{*}}$-measurable. We now conclude that $c_{m k}$ is $H^{1 / 4}\left(0, t^{*}\right)$ valued $\mathcal{F}_{t^{*}}$-measurable for $k=1, \cdots, m$. By the same argument as above, the measurability of $c_{m 0}(t)$ follows easily from its representation formula:

$$
c_{m 0}(t)=\left\langle\hat{u}_{1}, e_{0}\right\rangle+\int_{0}^{t} \int_{0}^{1} \xi_{m}\left(s, \omega, \sum_{j=1}^{m} c_{m j} \nabla e_{j}\right) e_{0} d x d s+B_{0}(t) .
$$


Next we establish a priori estimates.

Lemma 2.4. Let $u_{m}$ be defined by (2.5)-(2.8), and $\hat{u}_{0}, \hat{u}_{1}$ be given as in Theorem 1.2. Then, it holds that

$$
\begin{gathered}
\left\|\partial_{t} \nabla u_{m}\right\|_{L^{6}\left(\Omega ; L^{\infty}\left(0, T ; L^{2}(0,1)\right)\right)} \leq M \\
\left\|\partial_{t} \Delta u_{m}\right\|_{L^{6}\left(\Omega ; L^{2}\left(0, T ; L^{2}(0,1)\right)\right)} \leq M
\end{gathered}
$$

for some positive constants $M$ independent of $m$.

Proof. First we note that the quadratic variation of $B_{j}(t)$ defined by $(2.10)$ is

$$
\left\langle B_{j}, B_{k}\right\rangle_{t}=\int_{0}^{t} \int_{0}^{1}\left(\Phi^{\star} e_{j}\right)\left(\Phi^{\star} e_{k}\right) d x d s
$$

Let us fix $m$ and apply Ito's rule to the functional

$$
\left\|\partial_{t} \nabla u_{m}\right\|_{L^{2}(0,1)}^{2}=\sum_{k=1}^{m} \lambda_{k} c_{m k}^{2}(t) .
$$

It follows that for $P$-almost all $\omega \in \Omega$,

$$
\begin{aligned}
& \left\|\partial_{t} \nabla u_{m}(t)\right\|_{L^{2}(0,1)}^{2}-\left\|\partial_{t} \nabla u_{m}(0)\right\|_{L^{2}(0,1)}^{2} \\
& =-\int_{0}^{t} \int_{0}^{1} 2\left(\nabla \eta\left(\sum_{j=1}^{m} d_{m j} \nabla e_{j}, \sum_{j=1}^{m} c_{m j} \nabla e_{j}\right)\right) \partial_{t} \Delta u_{m} d x d s \\
& +\sum_{k=1}^{m} \int_{0}^{t} \int_{0}^{1} 2 \lambda_{k} c_{m k}\left(\Phi^{*} e_{k}\right) d W+\sum_{k=1}^{m} \int_{0}^{t} \int_{0}^{1} \lambda_{k}\left(\Phi^{\star} e_{k}\right)^{2} d x d s
\end{aligned}
$$

Here, by virtue of (2.1), it holds that for $P$-almost all $\omega$ and all $t$,

$$
\int_{0}^{1} \xi_{m}\left(t, \omega, \sum_{j=1}^{m} c_{m j} \nabla e_{j}\right) \partial_{t} \Delta u_{m} d x=0
$$

We will estimate the first integral in the right-hand side of (2.34). By means of (1.5) and (1.6), it is easy to see that for $P$-almost all $\omega \in \Omega$,

$$
\begin{aligned}
& -\int_{0}^{t} \int_{0}^{1} 2\left(\nabla \eta\left(\sum_{j=1}^{m} d_{m j} \nabla e_{j}, \sum_{j=1}^{m} c_{m j} \nabla e_{j}\right)\right) \partial_{t} \Delta u_{m} d x d s \\
& \leq-C_{1} \int_{0}^{t}\left\|\partial_{s} \Delta u_{m}(s)\right\|_{L^{2}(0,1)}^{2} d s+C_{2} \int_{0}^{t}\left\|\Delta u_{m}(s)\right\|_{L^{2}(0,1)}^{2} d s
\end{aligned}
$$


for some positive constants $C_{1}, C_{2}$ independent of $m, \omega$. Next we will estimate the second integral. Since it is a continuous martingale, we can use the BurkholderDavis-Gundy inequalities to find that for all $t \in[0, T]$,

$$
\begin{aligned}
& E\left(\sup _{s \in[0, t]}\left|\int_{0}^{s} \int_{0}^{1} \sum_{k=1}^{m} 2 \lambda_{k} c_{m k}\left(\Phi^{\star} e_{k}\right) d W\right|^{3}\right) \\
& \leq C_{3} E\left(\left(\int_{0}^{t} \int_{0}^{1}\left|\sum_{k=1}^{m} 2 \lambda_{k} c_{m k}\left(\Phi^{\star} e_{k}\right)\right|^{2} d x d s\right)^{3 / 2}\right) \\
& \leq C_{3} E\left(\left(\int_{0}^{t}\left(\sum_{k=1}^{m} 4 \lambda_{k} c_{m k}^{2}\right)\left(\sum_{k=1}^{m} \lambda_{k}\left\|\Phi^{\star} e_{k}\right\|_{L^{2}(0,1)}^{2}\right) d s\right)^{3 / 2}\right) \\
& \leq C_{3} E\left(\left(\int_{0}^{t} 4\left\|\partial_{s} \nabla u_{m}\right\|_{L^{2}(0,1)}^{2}\|\Phi\|^{2} d s\right)^{3 / 2}\right)
\end{aligned}
$$

for some positive constant $C_{3}$ independent of $m$. It follows from Hölder's inequality that for any $\epsilon>0$,

$$
E\left(\left(\int_{0}^{t}\left\|\partial_{s} \nabla u_{m}\right\|_{L^{2}(0,1)}^{2} d s\right)^{3 / 2}\right) \leq \epsilon E\left(\left(\int_{0}^{t}\left\|\partial_{s} \Delta u_{m}\right\|_{L^{2}(0,1)}^{2} d s\right)^{3}\right)+\frac{1}{\epsilon} .
$$

It is also easy to see that for $P$-almost all $\omega \in \Omega$ and all $t \in[0, T]$,

$$
\left\|\Delta u_{m}(t)\right\|_{L^{2}(0,1)}^{2} \leq 2\left\|\Delta u_{m}(0)\right\|_{L^{2}(0,1)}^{2}+2 T \int_{0}^{t}\left\|\partial_{s} \Delta u_{m}(s)\right\|_{L^{2}(0,1)}^{2} d s .
$$

Since $E\left(\left\|\Delta u_{m}(0)\right\|_{L^{2}(0,1)}^{6}+\left\|\partial_{t} \nabla u_{m}(0)\right\|_{L^{2}(0,1)}^{6}\right) \leq M$, for some positive constant $M$ independent of $m$, we can derive from (2.34) - (2.39)

$$
E\left(\left(\int_{0}^{t}\left\|\partial_{s} \Delta u_{m}\right\|_{L^{2}(0,1)}^{2} d s\right)^{3}\right) \leq C_{5}+C_{6} \int_{0}^{t} E\left(\left(\int_{0}^{s}\left\|\partial_{\sigma} \Delta u_{m}\right\|_{L^{2}(0,1)}^{2} d \sigma\right)^{3}\right) d s
$$

where $C_{5}$ and $C_{6}$ are positive constants independent of $m$. By Gronwall's inequality, it holds that

$$
E\left(\left(\int_{0}^{T}\left\|\partial_{t} \Delta u_{m}\right\|_{L^{2}(0,1)}^{2} d t\right)^{3}\right) \leq M
$$

for some positive constant $M$ independent of $m$. Combining this with (2.34) and (2.36) - (2.39), we find

$$
E\left(\sup _{t \in[0, T]}\left\|\partial_{t} \nabla u_{m}(t)\right\|_{L^{2}(0,1)}^{6}\right) \leq M
$$

for some positive constant $M$ independent of $m$. This completes the proof of Lemma 2.4 .

We will need some more regularity with respect to the time variable. This will be found with the help of the above estimates. First we recall some facts on interpolation. Let $\mathcal{U}$ be a Banach space and $\hat{L}^{\infty}(0, T ; \mathcal{U})$ be the completion of $\mathcal{U}$ valued simple functions in the sup-norm. Let $L^{\infty}(0, T ; \mathcal{U})$ be the usual space of $\mathcal{U}$-valued essentially bounded functions. If $\mathcal{U}$ is of infinite dimension, $\hat{L}^{\infty}(0, T ; \mathcal{U})$ is a proper closed subspace of $L^{\infty}(0, T ; \mathcal{U})$.

Lemma 2.5. The function class $\hat{L}^{\infty}\left(0, T ; L^{2}(0,1)\right) \cap L^{2}\left(0, T ; H^{1}(0,1)\right)$ is imbedded into $L^{6}\left(0, T ; H^{1 / 3}(0,1)\right)$. 
Proof. See Bergh and Löfström [3].

By virtue of the structure of each $u_{m}$, it follows from (2.41), (2.42) and Lemma 2.5 that

$$
\left\|\partial_{t} \nabla u_{m}\right\|_{L^{6}\left(\Omega ; L^{6}((0, T) \times(0,1))\right)} \leq M,
$$

for some positive constant $M$ independent of $m$. This yields

$$
\left\|\nabla u_{m}\right\|_{L^{6}\left(\Omega ; L^{6}((0, T) \times(0,1))\right)} \leq M,
$$

for some positive constant $M$ independent of $m$. Now we use (1.7) to obtain

$$
\left\|\eta\left(\nabla u_{m}, \partial_{t} \nabla u_{m}\right)\right\|_{L^{6 / 5}\left(\Omega ; L^{6 / 5}((0, T) \times(0,1))\right)} \leq M,
$$

for some positive constant $M$ independent of $m$. Since $H^{1 / 3}(0,1)$ is densely imbedded into $L^{6}(0,1), L^{6 / 5}(0,1)$ is imbedded into $H^{-1 / 3}(0,1)$, which can be characterized by

$$
H^{-1 / 3}(0,1)=\left\{\left.\sum_{k=1}^{\infty} a_{k} \sqrt{2} \sin (k \pi x)\left|\sum_{k=1}^{\infty} \lambda_{k}^{-1 / 3}\right| a_{k}\right|^{2}<\infty\right\} .
$$

Let $\Lambda_{m}$ denote the projection on $H^{-1 / 3}(0,1)$ defined by

$$
\Lambda_{m}\left(\sum_{k=1}^{\infty} a_{k} \sqrt{2} \sin (k \pi x)\right)=\sum_{k=1}^{m} a_{k} \sqrt{2} \sin (k \pi x)
$$

so that we can write

$$
\begin{aligned}
\sum_{k=1}^{m}\left(-\int_{0}^{1} \eta \nabla e_{k} d x\right) e_{k} & =\sum_{k=1}^{m}\left(\int_{0}^{1} \eta \sqrt{2 \lambda_{k}} \sin (k \pi x) d x\right) e_{k} \\
& =\nabla \sum_{k=1}^{m}\left(\int_{0}^{1} \eta \sqrt{2} \sin (k \pi x) d x\right) \sqrt{2} \sin (k \pi x) \\
& =\nabla\left(\Lambda_{m} \eta\left(\nabla u_{m}, \partial_{t} \nabla u_{m}\right)\right)
\end{aligned}
$$

But it follows from (2.45) and (2.47) that

$$
\left\|\nabla \Lambda_{m} \eta\left(\nabla u_{m}, \partial_{t} \nabla u_{m}\right)\right\|_{L^{6 / 5}\left(\Omega ; L^{6 / 5}\left(0, T ; H^{-4 / 3}(0,1)\right)\right)} \leq M,
$$

for some positive constant $M$ independent of $m$. It is easy to derive from (2.7) and (2.48) that for all $t \in[0, T]$,

$$
\begin{aligned}
\partial_{t} u_{m}(t)-\mathcal{P}_{m} \hat{u}_{1} & =\int_{0}^{t} \nabla\left(\Lambda_{m} \eta\left(\nabla u_{m}, \partial_{s} \nabla u_{m}\right)\right) d s \\
& +\int_{0}^{t} \mathcal{P}_{m} \xi_{m}\left(s, \omega, \partial_{s} \nabla u_{m}\right) d s \\
& +\Psi_{m}(t)
\end{aligned}
$$

where

$$
\Psi_{m}(t)=\sum_{k=0}^{m}\left(\int_{0}^{t} \int_{0}^{1} \Phi^{\star} e_{k} d W\right) e_{k}
$$


and $\mathcal{P}_{m}$ is the projection of $L^{2}(0,1)$ onto the subspace spanned by $\left\{e_{0}, \cdots, e_{m}\right\}$. Since $\left\{e_{k}\right\}_{k=0}^{\infty}$ is a complete orthonormal basis in $L^{2}(0,1)$, it is apparent that

$$
\begin{aligned}
E\left(\left\|\Psi_{m}\left(t_{2}\right)-\Psi_{m}\left(t_{1}\right)\right\|_{L^{2}(0,1)}^{2}\right) & =\sum_{k=0}^{m} E\left(\left|\int_{t_{1}}^{t_{2}} \int_{0}^{1} \Phi^{\star} e_{k} d W\right|^{2}\right) \\
& =E\left(\left|\int_{t_{1}}^{t_{2}} \sum_{k=0}^{m}\left\|\Phi^{\star} e_{k}\right\|_{L^{2}(0,1)}^{2} d t\right|\right) \\
& \leq M\left|t_{2}-t_{1}\right|,
\end{aligned}
$$

for some positive constant $M$ independent of $m, t_{1}, t_{2}$, which yields, for each $0<$ $\epsilon<1 / 2$,

$$
\int_{0}^{T} \int_{0}^{T} E\left(\frac{\left\|\Psi_{m}\left(t_{2}\right)-\Psi_{m}\left(t_{1}\right)\right\|_{L^{2}(0,1)}^{2}}{\left|t_{2}-t_{1}\right|^{1+2 \epsilon}}\right) d t_{1} d t_{2} \leq M_{\epsilon}
$$

for some positive constant $M_{\epsilon}$ independent of $m$. By taking $\epsilon=1 / 4$, we derive from $(2.53)$

$$
\left\|\Psi_{m}\right\|_{L^{2}\left(\Omega ; H^{1 / 4}\left(0, T ; L^{2}(0,1)\right)\right)} \leq M,
$$

for some positive constant $M$ independent of $m$. Next we derive from (1.8), (2.4) and $(2.30)$

$$
\left\|\mathcal{P}_{m} \xi_{m}\left(t, \omega, \partial_{t} \nabla u_{m}\right)\right\|_{L^{1}\left(\Omega ; L^{\infty}\left(0, T ; L^{2}(0,1)\right)\right)} \leq M,
$$

for some positive constant $M$ independent of $m$. Consequently, it follows from (2.49), (2.50), (2.54) and (2.55) that

$$
\left\|\partial_{t} u_{m}\right\|_{L^{1}\left(\Omega ; H^{1 / 4}\left(0, T ; H^{-4 / 3}(0,1)\right)\right)} \leq M,
$$

for some positive constant $M$ independent of $m$. Finally, we again use (1.8), (2.4), (2.29) and (2.30) to obtain

$$
\left\|\partial_{t} u_{m}\right\|_{L^{1}\left(\Omega ; L^{\infty}\left(0, T ; L^{2}(0,1)\right)\right)} \leq M
$$

for some positive constant $M$ independent of $m$. These estimates will be used to construct a pathwise solution.

2.2. Construction of a pathwise solution. We first observe the following fact.

Lemma 2.6. There is $\tilde{\Omega} \subset \Omega$ with $P(\tilde{\Omega})=1$ such that for each $\omega \in \tilde{\Omega}$,

$$
\begin{aligned}
& \int_{0}^{t} \int_{0}^{1} \Phi^{\star} e_{j} d W \quad \text { is continuous in } t \text { for all } e_{j} \text { 's, } \\
& \hat{u}_{0}(\cdot, \omega) \in \tilde{H}^{2}(0,1) \text { and } \hat{u}_{1}(\cdot, \omega) \in H^{1}(0,1), \\
& \xi(t, \omega, h) \text { and } \frac{\partial}{\partial h} \xi(t, \omega, h) \text { are continuous in }(t, h), \text { and } \\
& \frac{\partial}{\partial h} \xi(t, \omega, h) \text { is bounded, and }(2.7) \text { and }(2.8) \text { hold for all } \\
& t \in[0, T], \text { for every } m \text {. }
\end{aligned}
$$

From now on, $\tilde{\Omega}$ stands for the above subset. 
Lemma 2.7. For $0 \leq s<3 / 2, H^{s}(0,1)$ can be characterized by

$$
H^{s}(0,1)=\left\{\left.\sum_{k=0}^{\infty} a_{k} e_{k}|| a_{0}\right|^{2}+\sum_{k=1}^{\infty} \lambda_{k}^{s}\left|a_{k}\right|^{2}<\infty\right\} .
$$

Proof. See Fujiwara [7].

Lemma 2.8. Fix any $\omega \in \tilde{\Omega}$. Suppose that for $j=1,2, \partial_{t} v_{j} \in L^{2}\left(0, T ; \tilde{H}^{2}(0,1)\right) \cap$ $L^{6}\left(0, T ; H^{4 / 3}(0,1)\right), v_{j}(0)=\hat{u}_{0}(\cdot, \omega)$, and

$$
\begin{aligned}
\left\langle\partial_{t} v_{j}(t)-\hat{u}_{1}, e_{k}\right\rangle & =-\int_{0}^{t} \int_{0}^{1} \eta\left(\nabla v_{j}, \partial_{s} \nabla v_{j}\right) \nabla e_{k} d x d s \\
& +\int_{0}^{t} \int_{0}^{1} \xi\left(s, \omega, \partial_{s} \nabla v_{j}\right) e_{k} d x d s \\
& +\int_{0}^{t} \int_{0}^{1} \Phi^{\star} e_{k} d W
\end{aligned}
$$

holds for all $t \in[0, T]$ and all $e_{k}$ 's. Then, $v_{1}=v_{2}$.

Proof. Let us set

$$
v=v_{1}-v_{2}
$$

It follows from (2.62) that

$$
\begin{aligned}
\left\langle\partial_{t} v, e_{k}\right\rangle & =-\int_{0}^{t} \int_{0}^{1}\left(\eta\left(\nabla v_{1}, \partial_{s} \nabla v_{1}\right)-\eta\left(\nabla v_{2}, \partial_{s} \nabla v_{2}\right)\right) \nabla e_{k} d x d s \\
& +\int_{0}^{t} \int_{0}^{1}\left(\xi\left(s, \omega, \partial_{s} \nabla v_{1}\right)-\xi\left(s, \omega, \partial_{s} \nabla v_{2}\right)\right) e_{k} d x d s
\end{aligned}
$$

holds for all $t$ and all $e_{k}$ 's. Choose any $h \in H^{4 / 3}(0,1)$. Then, by Lemma 2.7,

$$
\sum_{k=0}^{N}\left\langle h, e_{k}\right\rangle e_{k} \rightarrow h \quad \text { strongly in } H^{4 / 3}(0,1), \text { as } N \rightarrow \infty .
$$

Since $H^{1 / 3}(0,1) \subset L^{6}(0,1), \partial_{t} v \in L^{2}\left(0, T ; \tilde{H}^{2}(0,1)\right)$, and

$$
\eta\left(\nabla v_{1}, \partial_{t} \nabla v_{1}\right)-\eta\left(\nabla v_{2}, \partial_{t} \nabla v_{2}\right) \in L^{6 / 5}((0, T) \times(0,1)),
$$

it follows from (2.64) and (2.65) that

$$
\begin{aligned}
\left\langle\partial_{t} v, h\right\rangle & =-\int_{0}^{t} \int_{0}^{1}\left(\eta\left(\nabla v_{1}, \partial_{s} \nabla v_{1}\right)-\eta\left(\nabla v_{2}, \partial_{s} \nabla v_{2}\right)\right) \nabla h d x d s \\
& +\int_{0}^{t} \int_{0}^{1}\left(\xi\left(s, \omega, \partial_{s} \nabla v_{1}\right)-\xi\left(s, \omega, \partial_{s} \nabla v_{2}\right)\right) h d x d s
\end{aligned}
$$

for almost all $t$ (with respect to the Lebesgue measure). Since the right-hand side is continuous in $t$, the left-hand side is also continuous and (2.66) holds for all $t$. We can infer from (2.66) that

$$
\begin{aligned}
\partial_{t}\left\langle\partial_{t} v, h\right\rangle & =-\int_{0}^{1}\left(\eta\left(\nabla v_{1}, \partial_{t} \nabla v_{1}\right)-\eta\left(\nabla v_{2}, \partial_{t} \nabla v_{2}\right)\right) \nabla h d x \\
& +\int_{0}^{1}\left(\xi\left(t, \omega, \partial_{t} \nabla v_{1}\right)-\xi\left(t, \omega, \partial_{t} \nabla v_{2}\right)\right) h d x
\end{aligned}
$$


holds in $L^{6 / 5}(0, T)$ for each $h \in H^{4 / 3}(0,1)$, and hence,

$$
\partial_{t t} v \in L^{6 / 5}\left(0, T ; H^{4 / 3}(0,1)^{*}\right),
$$

where $H^{4 / 3}(0,1)^{*}$ is the dual of $H^{4 / 3}(0,1)$. Since $\partial_{t} v \in L^{6}\left(0, T ; H^{4 / 3}(0,1)\right)$, it follows from (2.67) that

$$
\begin{aligned}
& e^{-\lambda t}\left\|\partial_{t} v(t)\right\|_{L^{2}(0,1)}^{2}=-\int_{0}^{t} \int_{0}^{1} \lambda e^{-\lambda s}\left(\partial_{s} v\right)^{2} d x d s \\
& -2 \int_{0}^{t} \int_{0}^{1} e^{-\lambda s}\left(\eta\left(\nabla v_{1}, \partial_{s} \nabla v_{1}\right)-\eta\left(\nabla v_{2}, \partial_{s} \nabla v_{2}\right)\right) \partial_{s} \nabla v d x d s \\
& +2 \int_{0}^{t} \int_{0}^{1} e^{-\lambda s}\left(\xi\left(s, \omega, \partial_{s} \nabla v_{1}\right)-\xi\left(s, \omega, \partial_{s} \nabla v_{2}\right)\right) \partial_{s} v d x d s
\end{aligned}
$$

for all $t \in[0, T]$. By considering (2.9) and (2.60), we can choose sufficiently large positive $\lambda$ in (2.69), which yields

$$
\int_{0}^{t} \int_{0}^{1}\left(\partial_{s} \nabla v\right)^{2} d x d s \leq K \int_{0}^{t} \int_{0}^{1}(\nabla v)^{2} d x d s
$$

for all $t \in[0, T]$ with some positive constant $K$. This, together with (2.69), yields that

$$
v=0
$$

on $[0, T]$, and the proof is complete.

We are now ready to construct a pathwise solution. Let us define

$$
\mathcal{X}=H^{1 / 4}\left(0, T ; H^{-4 / 3}(0,1)\right) \cap L^{\infty}\left(0, T ; H^{1}(0,1)\right) \cap L^{2}\left(0, T ; \tilde{H}^{2}(0,1)\right) .
$$

By virtue of (2.41), (2.42), (2.56) and (2.57), we have, for some positive constant $M$,

$$
\left\|\partial_{t} u_{m}\right\|_{L^{1}(\Omega ; \mathcal{X})} \leq M,
$$

for all $m$, which implies

$$
P\left(\left\{\left\|\partial_{t} u_{m}\right\|_{\mathcal{X}}>L\right\}\right) \leq M / L,
$$

for all $m$ and all $L>0$. Consequently, it is evident that

$$
P\left(\bigcap_{L=1}^{\infty} \bigcup_{k=1}^{\infty} \bigcap_{m=k}^{\infty}\left\{\left\|\partial_{t} u_{m}\right\|_{\mathcal{X}}>L\right\}\right)=0 .
$$

We now write

$$
\hat{\Omega}=\tilde{\Omega} \cap\left(\bigcup_{L=1}^{\infty} \bigcap_{k=1}^{\infty} \bigcup_{m=k}^{\infty}\left\{\left\|\partial_{t} u_{m}\right\|_{\mathcal{X}} \leq L\right\}\right),
$$

where $\tilde{\Omega}$ was chosen in Lemma 2.6. It follows from (2.75)

$$
P(\hat{\Omega})=1 \text {. }
$$

Fix any $\omega \in \hat{\Omega}$. Then, there is some $L \geq 1$ and a subsequence $\left\{u_{m_{\nu}}\right\}$ such that

$$
\left\|\partial_{t} v_{\nu}\right\|_{\mathcal{X}} \leq L
$$


for all $\nu \geq 1$, where $v_{\nu}=u_{m_{\nu}}$. For each $\nu \geq 1$,

$$
\begin{aligned}
& \left\langle\partial_{t} v_{\nu}(t)-\hat{u}_{1}, e_{k}\right\rangle=-\int_{0}^{t} \int_{0}^{1} \eta\left(\nabla v_{\nu}, \partial_{s} \nabla v_{\nu}\right) \nabla e_{k} d x d s \\
& +\int_{0}^{t} \int_{0}^{1} \xi_{m_{\nu}}\left(s, \omega, \partial_{s} \nabla v_{\nu}\right) e_{k} d x d s+\int_{0}^{t} \int_{0}^{1} \Phi^{\star} e_{k} d W
\end{aligned}
$$

for all $t \in[0, T]$ and for all $k=0, \cdots, m_{\nu}$. Also, we have

$$
\left\langle v_{\nu}(t)-\hat{u}_{0}, e_{k}\right\rangle=\int_{0}^{t}\left\langle\partial_{s} v_{\nu}, e_{k}\right\rangle d s
$$

for all $k=0, \cdots, m_{\nu}$. By Lemma 2.5 and (2.78), we can further extract a subsequence still denoted by $\left\{v_{\nu}\right\}$ such that for some function $v$ with $v(0)=\hat{u}_{0}(\cdot, \omega)$ and $\partial_{t} v \in \mathcal{X}$

$\nabla v_{\nu}$ and $\partial_{t} \nabla v_{\nu}$ converge to $\nabla v$ and $\partial_{t} \nabla v$, respectively, weakly in $L^{6}\left(0, T ; H^{1 / 3}(0,1)\right)$,

$\partial_{t} v_{\nu}$ converges to $\partial_{t} v$ strongly in $L^{2}\left(0, T ; H^{3 / 2}(0,1)\right)$,

(2.83) $\xi_{m_{\nu}}\left(t, \omega, \partial_{t} \nabla v_{\nu}\right)$ converges to $\xi\left(t, \omega, \partial_{t} \nabla v\right)$ weakly in $L^{2}((0, T) \times(0,1))$

and

(2.84) $\eta\left(\nabla v_{\nu}, \partial_{t} \nabla v_{\nu}\right)$ converges to $\eta\left(\nabla v, \partial_{t} \nabla v\right)$ weakly in $L^{6 / 5}((0, T) \times(0,1))$.

Here we have also used the following facts proved in Lions $[9]$.

Lemma 2.9. Let $g$ and $g_{k}$ 's, $k \geq 1$, belong to $L^{p}((0, T) \times(0,1)), 1<p<\infty$. Suppose that

$$
\left\|g_{k}\right\|_{L^{p}((0, T) \times(0,1))} \leq M, \quad \text { for some } M>0 \text { independent of } k,
$$

and, as $k \rightarrow \infty$,

$$
g_{k} \rightarrow g, \quad \text { for } \mu \text {-almost all }(t, x) .
$$

Then, $g_{k}$ converges to $g$ weakly in $L^{p}((0, T) \times(0,1))$, as $k \rightarrow \infty$.

Lemma 2.10. Suppose that $\mathcal{E}_{1}, \mathcal{E}_{2}$ and $\mathcal{E}_{3}$ are Hilbert spaces such that $\mathcal{E}_{1}$ is compactly embedded into $\mathcal{E}_{2}$ and $\mathcal{E}_{2}$ is embedded into $\mathcal{E}_{3}$. Then, $H^{1 / 4}\left(0, T ; \mathcal{E}_{3}\right) \cap$ $L^{2}\left(0, T ; \mathcal{E}_{1}\right)$ is compactly embedded into $L^{2}\left(0, T ; \mathcal{E}_{2}\right)$.

Next we fix any $k \geq 0$, and consider (2.79) for $m_{\nu} \geq k$. Multiply both sides of (2.79) by any $\psi(t) \in C_{0}^{\infty}((0, T))$ and integrate in $t$ over $(0, T)$. By passing $\nu \rightarrow \infty$ with help of (2.82) - (2.84), we arrive at

$$
\begin{aligned}
\int_{0}^{T} \psi(t)\left\langle\partial_{t} v(t)\right. & \left.-\hat{u}_{1}, e_{k}\right\rangle d t=\int_{0}^{T} \psi(t)\left(-\int_{0}^{t} \int_{0}^{1} \eta\left(\nabla v, \partial_{s} \nabla v\right) \nabla e_{k} d x d s\right. \\
& \left.+\int_{0}^{t} \int_{0}^{1} \xi\left(s, \omega, \partial_{s} \nabla v\right) e_{k} d x d s+\int_{0}^{t} \int_{0}^{1} \Phi^{\star} e_{k} d W\right) d t .
\end{aligned}
$$


This implies that

$$
\begin{aligned}
& \left\langle\partial_{t} v(t)-\hat{u}_{1}, e_{k}\right\rangle=-\int_{0}^{t} \int_{0}^{1} \eta\left(\nabla v, \partial_{s} \nabla v\right) \nabla e_{k} d x d s \\
& +\int_{0}^{t} \int_{0}^{1} \xi\left(s, \omega, \partial_{s} \nabla v\right) e_{k} d x d s+\int_{0}^{t} \int_{0}^{1} \Phi^{\star} e_{k} d W
\end{aligned}
$$

holds in the sense of distribution over $(0, T)$. Since the right-hand side is continuous in $t$, so is the left-hand side, and (2.88) holds for all $t \in[0, T]$ and for all $k \geq 0$. Since $v$ was constructed so that for the above fixed $\omega$,

$$
v(0)=\hat{u}_{0}(\cdot, \omega)
$$

it follows from Lemma 2.8 that $v$ is determined regardless of any particular subsequence. Thus, for each $\omega \in \hat{\Omega}$, we can choose $v$ unambiguously such that $\partial_{t} v \in \mathcal{X}$, and (2.88) and (2.89) hold. It remains to show that this $v$ is indeed a solution according to Definition 1.1.

Lemma 2.11. Suppose that $v=v(\cdot, \omega)$ has been constructed for each $\omega \in \hat{\Omega}$ according to the above procedure. Then, $v$ is a $H^{1}(0,1)$-valued continuous process adapted to $\left\{\mathcal{F}_{t}\right\}$.

Proof. For each $\omega \in \hat{\Omega}$, we have $\partial_{t} v \in \mathcal{X}$. Hence, $v(\cdot, \omega)$ is $\tilde{H}^{2}(0,1)$-valued continuous in $t \in[0, T]$ for each $\omega \in \hat{\Omega}$. Next we fix any arbitrary $t^{*} \in(0, T]$, and define

$$
\mathcal{X}_{*}=H^{1 / 4}\left(0, t^{*} ; H^{-4 / 3}(0,1)\right) \cap L^{\infty}\left(0, t^{*} ; H^{1}(0,1)\right) \cap L^{2}\left(0, t^{*} ; \tilde{H}^{2}(0,1)\right) .
$$

By virtue of Lemma 2.3 and the special structure of $\partial_{t} u_{m}$ given by (2.5) and (2.6), it is easy to see that each $\partial_{t} u_{m}$ is $\mathcal{X}_{*}$-valued $\mathcal{F}_{t^{*}}$-measurable and, for some positive constant $M$,

$$
\left\|\partial_{t} u_{m}\right\|_{L^{1}\left(\Omega ; \mathcal{X}_{*}\right)} \leq M
$$

for all $m$. Now we choose an arbitrary open ball $b_{r}(z)$ in $H^{1}(0,1)$, where $z$ is the center and $r>0$ is the radius. We will show that

$$
\begin{aligned}
& \hat{\Omega} \cap\left\{v\left(t^{*}\right) \in b_{r}(z)\right\} \\
& =\bigcup_{L=1}^{\infty} \bigcup_{n=1}^{\infty} \bigcap_{k=1}^{\infty} \bigcup_{m=k}^{\infty}\left(\left\{u_{m}\left(t^{*}\right) \in b_{r-1 / n}(z)\right\} \cap\left\{\left\|\partial_{t} u_{m}\right\|_{\mathcal{X}_{*}} \leq L\right\} \cap \hat{\Omega}\right),
\end{aligned}
$$

where $b_{r-1 / n}(z)$ means an empty set if $r \leq 1 / n$. Suppose $\omega^{*}$ belongs to the lefthand side. By recalling how $v$ was constructed at $\omega^{*}$, we find that there is a subsequence $\left\{u_{m_{\nu}}\right\}$ such that $u_{m_{\nu}}\left(t^{*}\right)$ converges strongly to $v\left(t^{*}\right)$ in $H^{1}(0,1)$ at $\omega^{*}$, and, for some integer $L \geq 1$,

$$
\left\|\partial_{t} u_{m_{\nu}}\right\|_{\mathcal{X}} \leq L
$$

for all $\nu$, and hence,

$$
\left\|\partial_{t} u_{m_{\nu}}\right\|_{\mathcal{X}_{*}} \leq L
$$

for all $\nu$. Consequently, $\omega^{*}$ belongs to the right-hand side. We next show the opposite inclusion. Let $\omega^{*}$ belong to the right-hand side. Then, there are $L^{*} \geq$ $1, n^{*} \geq 1$, and a subsequence $\left\{u_{m_{\nu}}\right\}$ such that

$$
u_{m_{\nu}}\left(t^{*}\right) \in b_{r-1 / n^{*}}(z), \quad \text { for all } \nu
$$


and

$$
\left\|\partial_{t} u_{m_{\nu}}\right\|_{\mathcal{X}_{*}} \leq L^{*}, \quad \text { for all } \nu .
$$

By Lemma 2.8 with $T$ replaced by $t^{*}$, we find that there is a subsequence $\left\{v_{j}\right\}$ of $\left\{u_{m_{\nu}}\right\}$ such that at $\omega^{*}$,

$$
v_{j}\left(t^{*}\right) \text { converges strongly to } v\left(t^{*}\right) \text { in } H^{1}(0,1),
$$

and

$$
v_{j}\left(t^{*}\right) \in b_{r-1 / n^{*}}(z), \quad \text { for all } j \geq 1 .
$$

Thus, $v\left(t^{*}\right) \in b_{r}(z)$ and $\omega^{*} \in \hat{\Omega} \cap\left\{v\left(t^{*}\right) \in b_{r}(z)\right\}$, and (2.92) has been established. Since each $u_{m}$ is a $H^{1}(0,1)$-valued continuous process adapted to $\left\{\mathcal{F}_{t}\right\}$, and $\mathcal{F}_{0}$ contains all $P$-negligible sets, $(2.92)$ implies that

$$
\left\{v\left(t^{*}\right) \in b_{r}(z)\right\} \in \mathcal{F}_{t^{*}} .
$$

Now the proof of Lemma 2.11 is complete.

Next we show that $v$ satisfies (1.12). Choose any $\phi \in C^{\infty}([0,1])$ with $\nabla \phi(0)=$ $\nabla \phi(1)=0$. Then, the sequence $\left\{\phi_{k}\right\}$ defined by

$$
\phi_{k}=\sum_{j=0}^{k}\left\langle\phi, e_{j}\right\rangle e_{j}
$$

converges to $\phi$ strongly in $\tilde{H}^{2}(0,1)$. Let us write

$$
\Psi_{k}(t)=\int_{0}^{t} \int_{0}^{1} \Phi^{\star} \phi_{k} d W, \quad k=0,1, \cdots
$$

and

$$
\Psi(t)=\int_{0}^{t} \int_{0}^{1} \Phi^{\star} \phi d W .
$$

All $\Psi_{k}$ 's and $\Psi$ are continuous martingales, and it follows from the BurkholderDavis-Gundy inequalities that

$$
E\left(\sup _{t \in[0, T]}\left|\Psi_{k}(t)-\Psi(t)\right|^{2}\right) \leq \int_{0}^{T}\left\|\Phi^{\star}\left(\phi_{k}-\phi\right)\right\|_{L^{2}(0,1)}^{2} d t .
$$

Hence, there is a subsequence still denoted by $\left\{\Psi_{k}(t)\right\}$ such that

$$
\sup _{t \in[0, T]}\left|\Psi_{k}(t)-\Psi(t)\right| \rightarrow 0, \quad \text { for } P \text {-almost all } \omega .
$$

By (2.88), we have

$$
\begin{aligned}
& \left\langle\partial_{t} v(t)-\hat{u}_{1}, \phi_{k}\right\rangle=-\int_{0}^{t} \int_{0}^{1} \eta\left(\nabla v, \partial_{s} \nabla v\right) \nabla \phi_{k} d x d s \\
& +\int_{0}^{t} \int_{0}^{1} \xi\left(s, \omega, \partial_{s} \nabla v\right) \phi_{k} d x d s+\int_{0}^{t} \int_{0}^{1} \Phi^{\star} \phi_{k} d W
\end{aligned}
$$

for all $k \geq 0$, all $t \in[0, T]$ and all $\omega \in \hat{\Omega}$. Since $\eta\left(\nabla v, \partial_{t} \nabla v\right) \in L^{6 / 5}((0, T) \times(0,1))$ for each $\omega \in \hat{\Omega}$, it is evident that as $k \rightarrow \infty$,

$$
\int_{0}^{t} \int_{0}^{1} \eta\left(\nabla v, \partial_{s} \nabla v\right) \nabla \phi_{k} d x d s \rightarrow \int_{0}^{t} \int_{0}^{1} \eta\left(\nabla v, \partial_{s} \nabla v\right) \nabla \phi d x d s
$$


strongly in $C([0, T])$, for each $\omega \in \hat{\Omega}$. Also, as $k \rightarrow \infty$,

$$
\int_{0}^{t} \int_{0}^{1} \xi\left(s, \omega, \partial_{s} \nabla v\right) \phi_{k} d x d s \rightarrow \int_{0}^{t} \int_{0}^{1} \xi\left(s, \omega, \partial_{s} \nabla v\right) \phi d x d s
$$

strongly in $C([0, T])$, for each $\omega \in \hat{\Omega}$. Meanwhile, since $\partial_{t} v \in L^{\infty}\left(0, T ; H^{1}(0,1)\right)$ for each $\omega \in \hat{\Omega}$, it follows that

$$
\left\langle\partial_{t} v(t)-\hat{u}_{1}, \phi_{k}\right\rangle \rightarrow\left\langle\partial_{t} v(t)-\hat{u}_{1}, \phi\right\rangle,
$$

as $k \rightarrow \infty$, strongly in $L^{\infty}(0, T)$ for each $\omega \in \hat{\Omega}$. By virtue of (2.105), we find that $\left\langle\partial_{t} v(t)-\hat{u}_{1}, \phi_{k}\right\rangle \in C([0, T])$, for all $k$, and hence, by (2.108),

$$
\left\langle\partial_{t} v(t)-\hat{u}_{1}, \phi\right\rangle \in C([0, T]) .
$$

It now follows from $(2.104)-(2.108)$ that

$$
\begin{aligned}
& \left\langle\partial_{t} v(t)-\hat{u}_{1}, \phi\right\rangle=-\int_{0}^{t} \int_{0}^{1} \eta\left(\nabla v, \partial_{s} \nabla v\right) \nabla \phi d x d s \\
& +\int_{0}^{t} \int_{0}^{1} \xi\left(s, \omega, \partial_{s} \nabla v\right) \phi d x d s+\int_{0}^{t} \int_{0}^{1} \Phi^{\star} \phi d W
\end{aligned}
$$

holds for all $t \in[0, T]$, for $P$-almost all $\omega$. This completes the proof of existence of a solution. If there are two solutions $v_{1}$ and $v_{2}$, we can use the same argument as in Lemma 2.8 to find that $v_{1}=v_{2}$ for $P$-almost all $\omega$. This ends the proof of our main result.

Remark. We could also include a random perturbation of the form $\xi\left(t, \omega, u_{x}\right)$ if $\xi$ satisfies the conditions [II] and [III] above with (1.8) replaced by

$$
\left\{\begin{array}{l}
\xi(t, \omega, h) \in L^{6}\left(\Omega ; L^{\infty}((0, \infty) \times R)\right), \\
\frac{\partial \xi}{\partial h}(t, \omega, h) \in L^{6 / 5}\left(\Omega ; L^{\infty}((0, \infty) \times R)\right) .
\end{array}\right.
$$

Under these conditions, the perturbation may look very mild, but the monotonicity is essentially broken by randomness of $L^{p}\left(\Omega ; L^{\infty}((0, \infty) \times R)\right)$ because $p<\infty$. If $p=\infty$, randomness does not cause any obstacle. We also note that the Dirichlet boundary conditions can be handled by the same procedure. But (1.8) should be replaced by (2.111).

\section{REFERENCES}

[1] Andrews, G., On the existence of solutions to the equation $u_{t t}=u_{x x t}+\sigma\left(u_{x}\right)_{x}$, J. Diff. 35 (1980), pp. 200 - 231. MR 81d:35073

[2] Bensoussan, A. and Temam, R., Équations aux dérivées partielles stochastiques non linéaires, Israel J. Math. 11 (1972), pp. 95 - 129. MR 56:6867

[3] Bergh, J. and Löfström, J., Interpolation Spaces, Springer-Verlag, Berlin-Heidelberg-New York, 1976. MR 58:2349

[4] Dafermos, C.M., The mixed initial-boundary value problem for the equations of nonlinear one-dimensional viscoelasticity, J. Diff. Equations 6 (1969), pp. 71 - 86. MR 39:3168

[5] Da Prato, G. and Zabczyk, J., Stochastic Equations in Infinite Dimensions, Cambridge University Press, 1992. MR 95g:60073

[6] de Bouard, A. and Debussche, A., On the stochastic Korteweg-de Vries equation, J. Func. Analysis 154 (1998), pp. 215 - 251. MR 99c:35209

[7] Fujiwara, D., Concrete characterization of the domains of fractional powers of some elliptic differential operators of the second order, Proc. Japan Acad. 43 (1967), pp. 82 - 86. MR 35:7170 
[8] Greenberg, J.M., MacCamy, R.C. and Mizel, V.J., On the existence, uniqueness and stability of solutions of the equation $\sigma^{\prime}\left(u_{x}\right) u_{x x}+\lambda u_{x t x}=\rho_{0} u_{t t}$, J. Math. Mech. 17 (1968), pp. 707 - 728. MR 37:623

[9] Lions, J.L., Quelques méthodes de résolution des problémes aux limites non linéaires, Dunod, Paris, 1969. MR 41:4326

[10] Métivier, M. and Pistone, G., Sur une équation d'évolution stochastique, Bull. Soc. Math. France 104 (1976), pp. 65 - 85. MR 54:8866

[11] Métivier, M. and Viot, M., On weak solutions of stochastic partial differential equations, Lecture Notes in Math. No.1322, Springer-Verlag, 1987, pp. 139 - 150. MR 90a:60113

[12] Pardoux, E., Sur des équations aux dérivées partielles stochastiques monotones, C.R.Acad. Sci. Paris, série A 275 (1972), pp. 101 - 103. MR 47:1129

[13] Printems, J., The stochastic Korteweg-de Vries equation in $L^{2}(R)$, J. Diff. Equations 153 (1999), pp. 338 - 373. MR 2001g:35280

[14] Viot, M., Solution en loi d'une équation aux dérivées partiellles stochastique non linéaire: méthode de compacité, C.R. Acad. Sci. Paris, série A 278 (1974), pp. 1185 - 1188. MR 50:7824a

[15] Viot, M., Solution en loi d'une équation aux dérivées partiellles stochastique non linéaire: méthode de monotonie, C.R. Acad. Sci. Paris, série A 278 (1974), pp. 1405 - 1408. MR 50:7824b

[16] Viot, M., Solutions faibles aux équations aux dérivées partielles stochastiques non linéaires, Thèse, Université Pierre et Marie Curie, Paris (1976).

[17] Walsh, J.B., An introduction to stochastic partial differential equations, Lecture Notes in Math., No.1180, Springer-Verlag, 1984, pp. 266 - 437. MR 88a:60114

[18] Yamada, T. and Watanabe, S., On the uniqueness of solutions of stochastic differential equations, J. Math. Kyoto Univ. 11 (1971), pp. 155 - 167, pp. 553 - 563. MR 43:4150 MR 44:6071

Department of Mathematics, Virginia Tech, Blacksburg, Virginia 24061-0123

E-mail address: kim@math.vt.edu 